\title{
Real-World Insurance Claims Analysis of Venous Thromboembolism in Japanese Patients with Inflammatory Bowel Disease
}

\author{
Mikihiro Fujiya ${ }^{1}$ (1) $\cdot$ Tsutomu Kawaguchi $^{2} \cdot$ Shoko Arai $^{2} \cdot$ Naoki Isogawa $^{3} \cdot$ Shintaro Hiro $^{3}$. Fumihiro Matsumoto ${ }^{2}$. \\ Satoshi Yamaguchi ${ }^{2,4} \cdot$ Noritoshi Yoshii $^{2} \cdot$ Mashio Nakamura ${ }^{5} \cdot K^{2}$ atsuyoshi Matsuoka ${ }^{6}$
}

Received: 23 February 2021 / Accepted: 13 January 2022 / Published online: 12 February 2022

(c) The Author(s) 2022

\begin{abstract}
Background Inflammatory bowel disease (IBD), which encompasses both ulcerative colitis (UC) and Crohn's disease (CD), is a risk factor for venous thromboembolism (VTE), which includes deep vein thrombosis (DVT) and pulmonary embolism (PE).

Aim To investigate the incidence of, and risk factors for, VTE in patients with IBD in Japan.

Methods This was a retrospective, non-interventional study in patients with IBD from the Japan Medical Data Center claims database. Incidence rates (IRs; unique patients with events per 100 patient-years) were calculated for VTE, DVT, and PE among the IBD, UC, and CD cohorts. Odds ratios of potential risk factors were calculated by univariate and multivariate analyses in a nested case-control design.

Results Overall, 16273 patients with IBD were included: 13585 with UC and 3443 with CD. VTE events occurred in 1.3\%, $1.2 \%$, and $1.9 \%$ of patients with IBD, UC, and CD, respectively. In patients with IBD, UC, and CD, IRs of VTE were 0.45 , 0.40 , and 0.64 , respectively, IRs of DVT were $0.42,0.38$, and 0.61 , respectively, and IRs of PE were 0.07, 0.07, and 0.11, respectively. In patients with IBD, treatment history (immunomodulators), cardiovascular risk (hypertension, high-density lipoprotein or diabetes mellitus, and history of coronary artery disease or heart failure), malignancy, and undergoing major surgery were identified as potential risk factors for VTE in the multivariate analysis, with similar risk factors reported for patients with UC and CD.
\end{abstract}

Conclusions This large study provides insight into the incidence and risk factors for VTE in patients with IBD from Japan.

Keywords Incidence $\cdot$ Inflammatory bowel diseases $\cdot$ Japan $\cdot$ Risk factors $\cdot$ Venous thromboembolism

Mikihiro Fujiya

fjym@asahikawa-med.ac.jp

Tsutomu Kawaguchi

t-kwgch@koto.kpu-m.ac.jp

Shoko Arai

Shoko.Arai@pfizer.com

Naoki Isogawa

alljno1bands626@yahoo.co.jp

Shintaro Hiro

shintaro.hiro@pfizer.com

Fumihiro Matsumoto

fumihiro.matsumoto@pfizer.com

Satoshi Yamaguchi

satoshi.yamaguchi@pfizer.com

Noritoshi Yoshii

yoshiinoritoshi@gmail.com

Mashio Nakamura

mashio@clin.medic.mie-u.ac.jp
Katsuyoshi Matsuoka

matsuoka.katsuyoshi@gmail.com

1 Division of Gastroenterology and Hematology/Oncology, Department of Medicine, Asahikawa Medical University, 2-1 Midorigaoka-Higashi, Asahikawa 078-8510, Japan

2 Immunology \& Inflammation Medical Affairs, Pfizer Japan Inc, Shinjuku Culture Quint Bldg 3-22-7 Yoyogi Shibuya-ku, Tokyo 151-8589, Japan

3 Biometrics \& Data Management, Pfizer R\&D Japan, Shinjuku Culture Quint Bldg 3-22-7 Yoyogi Shibuya-ku, Tokyo 151-8589, Japan

4 International University of Health and Welfare, Tokyo, Japan

5 Department of Internal Medicine, Pediatrics and Cardiology, Hidamarinooka Nakamura Medical Clinic, 7-1510 Hidamarinooka, Kuwana 511-0867, Japan

6 Division of Gastroenterology and Hepatology, Department of Internal Medicine, Toho University Sakura Medical Center, Sakura City, Chiba 285-0841, Japan 


\section{Introduction}

Inflammatory bowel disease (IBD) encompasses ulcerative colitis (UC), which is characterized by diffuse inflammation of the mucosa of the colon, and Crohn's disease (CD), which is characterized by transmural inflammation of any part of the gastrointestinal tract [1]. The prevalence of UC and CD in Japan has been reported to have increased from 0.05 and 0.02 per 100 persons in 2003 [2] to 0.17 and 0.06 per 100 persons in 2014, respectively [3]. IBD is a known risk factor for venous thromboembolism (VTE), including deep vein thrombosis (DVT) and pulmonary embolism (PE) [4], with the risk increasing further during an IBD flare or hospitalization $[5,6]$. DVT is defined as the formation of a blood clot in a deep vein; while PE is a life-threatening complication that occurs when part of the blood clot travels to the heart and lungs, resulting in a blockage [7]. Multiple risk factors for VTE in patients with IBD have been identified, including inflammatory activity, hospitalization, surgery, pregnancy, older age, a diagnosis of UC, disease phenotype, and drug therapy (particularly steroids) [6, 8-11]. Due to the higher incidence of VTE in patients with IBD, prophylactic treatments for VTE have been recommended for inpatients and should be considered for outpatients who have risk factors, such as recent surgery and active disease, in European countries [12].

The incidence of VTE may differ by race and ethnicity [13], with reported incidence rates (IRs; unique patients with events per 100 patient-years) in the Western countries of Denmark and Canada ranging from 0.14 to 0.30 for DVT and from 0.10 to 0.20 for PE in patients with UC, and from 0.13 to 0.31 and from 0.10 to 0.11 , respectively, in patients with CD $[14,15]$. Sonoda et al. investigated 47 consecutive Japanese patients who were hospitalized with IBD and concluded that $17.0 \%$ had VTE during the study period [16]. Additionally, a previous retrospective cohort study conducted in a single hospital in Japan, which included 340 patients who were hospitalized with IBD, reported that the incidence of VTE in patients with UC was $16.9 \%$ during the study period; this was significantly higher than the incidence in patients with $\mathrm{CD}(3.6 \%)$ [17]. Furthermore, Weng et al. investigated 3178 Taiwanese patients with IBD and found that the IRs of DVT were 0.07 and 0.18 in patients with UC and $\mathrm{CD}$, respectively, and the IRs of PE were 0.04 and 0.03 in patients with UC and CD, respectively [18]. However, the sample sizes of these studies in Asian populations may not be sufficient to accurately determine the incidence of, and risk factors for, IBD-associated VTE.

In this study, we utilized a large, health insurance-based claims database to determine the incidence of, and risk factors for, VTE, including DVT and PE, in Japanese patients with IBD.

\section{Methods}

\section{Study Design and Patients}

This was a retrospective, non-interventional study in patients with a diagnosis of IBD (International Classification of Diseases, 10th revision [ICD-10] code) extracted from the Japan Medical Data Center (JMDC) claims database, a health insurance-based claims database in Japan. The JMDC claims database is an epidemiological receipt database that has accumulated receipts (inpatient, outpatient, and dispensing) received from multiple employees' health insurance associations since 2005, and includes a cumulative observed population of 3 million patients. JMDC can track individual patient claims data across clinics, hospitals, and dispensing pharmacies.

Patients were required to have a definitive diagnosis of $\mathrm{UC}$ or $\mathrm{CD}$, either in inpatient or outpatient claims, using extended ICD-10 coding for K51 (UC) or K50, M07, and M09 (CD) between June 2013 and May 2018. In addition, within the same claim month or \pm 1 claim month after the first definitive diagnosis of UC or CD, patients were required to have at least one of: prescription record for systemic agent (biological therapy [includes tumor necrosis factor inhibitors (TNFi) infliximab, adalimumab, golimumab (UC only) and ustekinumab (CD only)], immunomodulators/immunosuppressants [includes 5-aminosalicylates (5-ASA) sulfasalazine, sulphapyridine, mesalazine, and tacrolimus, azathioprine, 6-mercaptopurine, and cyclosporine (UC only)], corticosteroids [includes prednisolone, budesonide, betamethasone, and methylprednisolone], antidiarrheal treatment [includes loperamide hydrochloride (UC only)], antibiotics [includes ciprofloxacin and metronidazole (CD only)]; record of use of leukocytapheresis treatment; or diagnosis of postoperative (UC: colostomy, UC postoperative, total colectomy; CD: strictureplasty, ileostomy, colostomy, bowel resection, colectomy, CD postoperative, total colectomy, perianal abscess, anal fistula) in either inpatient or outpatient claims. The claims-based definition of UC has been validated in a study with relatively high positive predictive value. Antidiarrheal treatment was included in the definition of patients with UC in order to capture post-/ peri-operative patients, as the validation study showed that these patients, particularly those who received total colectomy, could be identified using the algorithm of 'disease name and antidiarrheal drugs' with good positive predictive value (unpublished data).

Index date was defined as: the date of initial prescription record for systemic agent (biological therapy, immunomodulatory/immunosuppressant, corticosteroids, antidiarrheal treatment [UC], antibiotics [CD]); record of use of 
leukocytapheresis treatment; or diagnosis of postoperative in either inpatient or outpatient claims, following the first record of $\mathrm{UC}$ or $\mathrm{CD}$ diagnosis. IBD, $\mathrm{UC}$, and $\mathrm{CD}$ cohorts were assessed. In the case that a patient met both UC and CD cohort definitions, the index date for the IBD cohort was defined as the earliest of the index dates. Patients with a claim diagnosis code of Behçets disease within the same claim month or \pm 1 claim month were excluded. The study follow-up period commenced at the index date and ended at lost medical or pharmacy coverage, or end of study (May 2018), whichever was earlier. The exposure period was from the index date to the date of the first event for patients with the event of VTE, censored at the end of the follow-up period for patients without the event, or end of study (May 2018), whichever was earlier; drug discontinuation was not considered.

\section{Assessments}

Comprehensive medical information was retrieved from the claims database. The fundamental definition of VTE was a claim diagnosis code of VTE (ICD-10) with any of the following seven events within the same claim month or \pm 1 claim months of the claim diagnosis code of VTE: prescription of heparin, prescription of fondaparinux, inferior vena cava filter placement, thrombus removal, prescription of urokinase, prescription of tissue plasminogen activator, or prescription of oral anticoagulant. A VTE event was defined as a fundamental definition of VTE after index date, without any claim medical code of the above seven events for at least 2 months prior to the fundamental definition of VTE. A washout period of at least 2 months was set for VTE events to enable the new VTE event to be captured, and to exclude patients with VTE who had received maintenance therapy for thrombosis.
Table 1 Baseline characteristics of patients with IBD, UC, and $\mathrm{CD}$ in the retrospective cohort study

\begin{tabular}{|c|c|c|c|}
\hline & $\begin{array}{l}\text { IBD } \\
N=16273\end{array}$ & $\begin{array}{l}\mathrm{UC} \\
N=13585\end{array}$ & $\begin{array}{l}\mathrm{CD} \\
N=3443\end{array}$ \\
\hline Female, $n(\%)$ & $5894(36.2)$ & $5297(39.0)$ & $813(23.6)$ \\
\hline \multicolumn{4}{|l|}{ Age, years } \\
\hline Mean (SD) & $39.9(13.1)$ & $40.8(13.0)$ & $35.6(13.1)$ \\
\hline Median (range) & $40.0(0-74)$ & $41.0(0-74)$ & $35.0(0-74)$ \\
\hline History of VTE, $n(\%)$ & $52(0.3)$ & $38(0.3)$ & $18(0.5)$ \\
\hline \multicolumn{4}{|l|}{ VTE event, $n(\%)$} \\
\hline Overall & $215(1.32)$ & $160(1.18)$ & 65 (1.89) \\
\hline Inpatient $^{\dagger}$ & $167(77.7)$ & $122(76.3)$ & $53(81.5)$ \\
\hline Outpatient $^{\dagger}$ & $48(22.3)$ & $38(23.8)$ & $12(18.5)$ \\
\hline \multicolumn{4}{|l|}{ Time to VTE event, $\stackrel{ }{\ddagger}$ days } \\
\hline Mean (SD) & $579.6(548.7)$ & $564.7(536.0)$ & $589.1(573.8)$ \\
\hline Median (range) & $401.0(1-1868)$ & $399.0(1-1860)$ & $365.0(1-1868)$ \\
\hline \multicolumn{4}{|c|}{ Severe VTE event, ${ }^{\S} n(\%)$} \\
\hline Overall & $6(0.04)$ & $6(0.04)$ & $2(0.06)$ \\
\hline Inpatient ${ }^{\mathbb{I}}$ & $6(100.0)$ & $6(100.0)$ & $2(100.0)$ \\
\hline Outpatient $^{\text {II }}$ & $0(0.00)$ & $0(0.00)$ & $0(0.00)$ \\
\hline \multicolumn{4}{|l|}{ Time to follow-up, days } \\
\hline Mean (SD) & $1081.2(625.6)$ & $1071.4(623.5)$ & $1085.4(624.9)$ \\
\hline Median (range) & $1096.0(1-1918)$ & $1096.0(1-1918)$ & $1096.0(1-1918)$ \\
\hline
\end{tabular}

${ }^{\dagger}$ Denominator is the number of patients with VTE

†Time to VTE event was only captured for patients who had a VTE event and was defined as the time to a fundamental definition of VTE following the index date

${ }^{\S}$ Patients with VTE who received invasive treatment, including inferior vena cava filter placement or thrombus removal, from the event date until the study end

IDenominator is the number of patients with severe VTE

$C D$ Crohn's disease, $I B D$ inflammatory bowel disease, $N$ number of patients in the analysis population, $n$ number of patients in the category, $S D$ standard deviation, $U C$ ulcerative colitis, VTE venous thromboembolism 


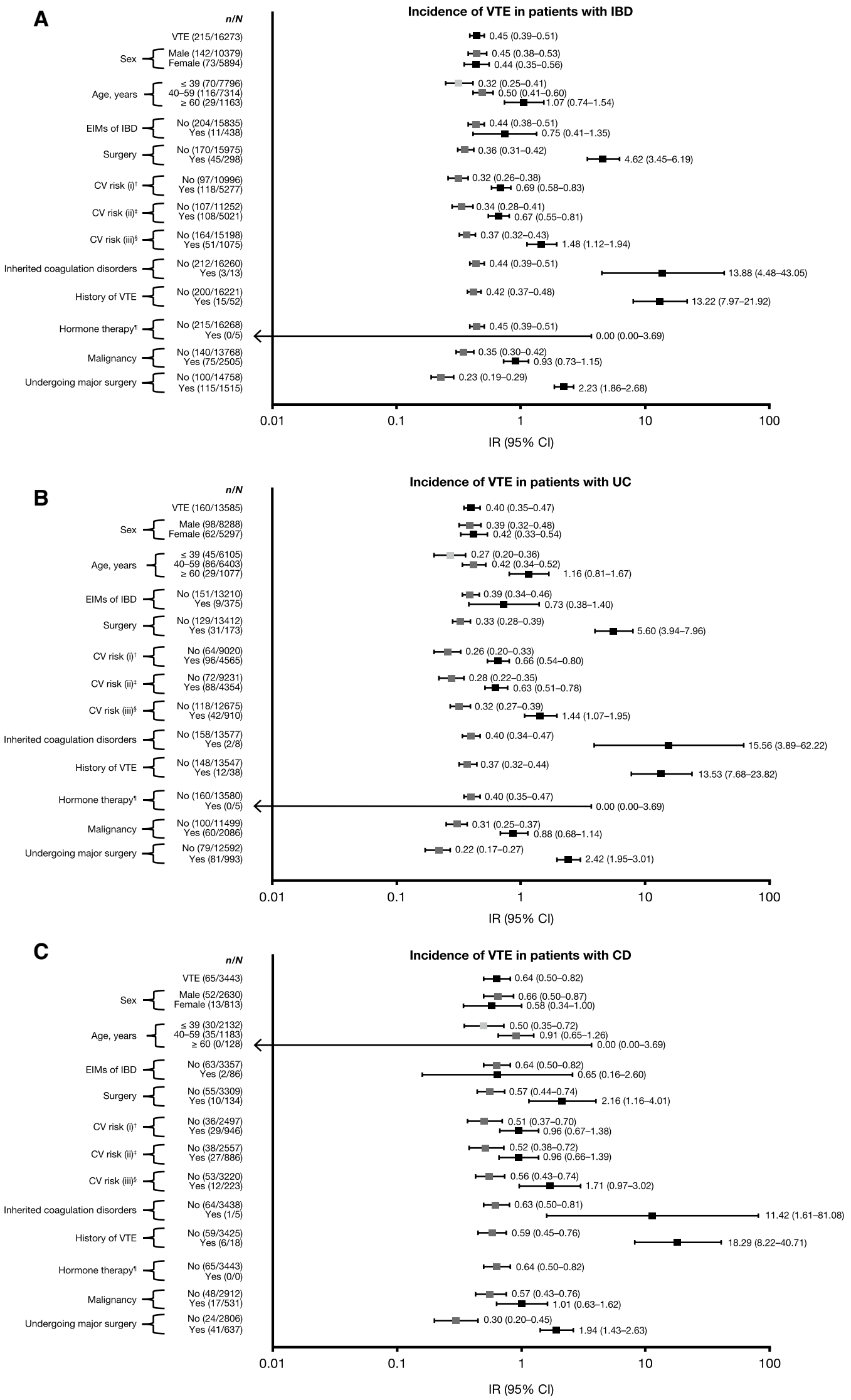


४Fig. 1 Incidence of VTE in patients with $\mathbf{a}$ IBD, $\mathbf{b}$ UC, and $\mathbf{c}$ CD by potential risk factors in the retrospective cohort study. ${ }^{\dagger} \mathrm{CV}$ risk (i): hypertension, high-density lipoprotein, diabetes mellitus, history of coronary artery disease, heart failure. ${ }^{\ddagger} \mathrm{CV}$ risk (ii): hypertension, high-density lipoprotein, diabetes mellitus. ${ }^{\S} \mathrm{CV}$ risk (iii): history of coronary artery disease, heart failure. "Hormone therapy: oral contraceptive, hormone replacement therapy. $C D$ Crohn's disease, $C I$ confidence interval, $C V$ cardiovascular, EIM extraintestinal manifestation, $I B D$ inflammatory bowel disease, $I R$ incidence rate (unique patients with events per 100 patient-years), $N$ number of patients in the analysis population, $n$ number of patients in the category, $U C$ ulcerative colitis, VTE venous thromboembolism

\section{Statistical Analyses}

\section{Retrospective Cohort Study}

Patient demographics and clinical information/events, concordance rate, and bed counts of medical facility were summarized. The concordance rate of medical facility was calculated by determining the degree to which the facility at index and the facility where the VTE event was identified for each patient matched. The bed count of each medical facility was determined for each patient at index and during a VTE event.

IRs with 95\% confidence intervals (CIs) for VTE, DVT, and PE among the IBD, UC, and CD cohorts were calculated. IRs were also calculated by the following potential risk factors: sex, age ( $\leq 39 / 40-59 / \geq 60$ years), history of VTE, extraintestinal manifestations (EIMs) of IBD, surgery, treatment history (5-ASA, steroids, immunomodulators, TNFi, central venous catheter), cardiovascular (CV) risk (hypertension, high-density lipoprotein, diabetes mellitus, history of coronary artery disease, heart failure), inherited coagulation disorders, hormone therapy, malignancy, and undergoing major surgery (Table S1).

\section{Nested Case-Control Study}

For each VTE patient with IBD, four control patients were randomly selected from the non-VTE patients with IBD and matched by period from the index date to VTE onset, to investigate the possible risk factors for VTE. Baseline clinical variables were summarized. Using logistic regression, the odds ratios (ORs; with $95 \%$ CI) of potential risk factors were calculated in univariate and multivariate analyses (Table S1). Multivariate analysis only included the variables with $\mathrm{OR} \leq 0.67$ or $\geq 1.5$ (point estimation) in univariate analysis. The above analyses were performed in the IBD, UC, and CD cohorts.

\section{Compliance with Ethical Standards}

The study was conducted in accordance with legal and regulatory requirements, as well as with scientific purpose, value, and rigor, and followed generally accepted research practices described in the Guidelines for Good Pharmacoepidemiology Practices (GPP) issued by the International Society for Pharmacoepidemiology (ISPE), Good Epidemiological Practice (GEP) guidelines issued by the International Epidemiological Association (IEA), and Good Practices for Outcomes Research issued by the International Society for Pharmacoeconomics and Outcomes Research (ISPOR).

\section{Results}

\section{Retrospective Cohort Study}

\section{Patients}

Overall, 16273 patients with IBD were included in this study (13 585 with UC and 3443 with CD), of whom $0.3 \%, 0.3 \%$, and $0.5 \%$, respectively, had a history of VTE. The majority of patients were male $(63.8 \%$ of patients with IBD, $61.0 \%$ of patients with UC, and $76.4 \%$ of patients with CD), with a mean age of 39.9, 40.8, and 35.6 years, respectively. Of these, $1.3 \%$ of patients with IBD, $1.2 \%$ of patients with UC, and $1.9 \%$ of patients with CD experienced a VTE event, with $0.04 \%, 0.04 \%$, and $0.06 \%$ of patients, respectively, experiencing a severe VTE event that required invasive treatment, including inferior vena cava filter placement or thrombus removal. The majority of VTE events occurred in inpatients and all patients with severe VTE events were inpatients. The mean follow-up time was 3.0, 2.9, and 3.0 years in the IBD, $\mathrm{UC}$, and CD cohorts, respectively, with an average time of 1.6, 1.5, and 1.6 years, respectively, until patients experienced a VTE event (Table 1). In the IBD and UC cohorts, the highest proportions of patients $(35.6 \%$ and $39.0 \%$, respectively) attended small-scale facilities (0-19 beds) at index; for the CD cohort, the highest proportion of patients $(36.8 \%)$ attended large-scale facilities $(500+$ beds $)$ at index. For all cohorts, the majority of patients (72.5-86.2\%) attended large-scale facilities $(500+$ beds $)$ during a VTE event. In addition, for all cohorts, the concordance rate of medical facility of the index disease and that of the VTE event was almost 50\% (Table S2).

\section{IRs of VTE, PE, and DVT by Risk Factor Subgroups in the IBD, UC, and CD Cohorts}

In patients with IBD, UC, and $\mathrm{CD}$, the overall IRs of VTE were 0.45 (95\% CI 0.39-0.51), 0.40 (0.35-0.47), and 0.64 (0.50-0.82), respectively, IRs of DVT were $0.42(0.37-0.48)$, $0.38(0.32-0.44)$, and $0.61(0.48-0.79)$, respectively, and IRs of PE were 0.07 (0.05-0.10), 0.07 (0.04-0.10), and 0.11 (0.06-0.19), respectively. 
When analyzed by hospitalization status, the proportion of inpatients with IBD, UC, and CD with VTE events were $77.7 \%, 76.3 \%$, and $81.5 \%$, respectively (for patients with multiple events, only the first event was counted). The proportion of inpatients with IBD, UC, and CD with DVT events were $77.0 \%, 75.8 \%$, and $81.7 \%$, and with PE events were $70.6 \%, 64.0 \%$, and $81.8 \%$, respectively (for patients with multiple events, only the first event was counted).

The impact of potential risk factors and treatment history on the incidence of VTE in the IBD, UC, and CD cohorts is presented in Fig. 1 and Fig. 2, respectively. Corresponding analyses showing the impact of these factors on the incidence of DVT and PE are shown in Fig. S1 and Fig. S2. It should be noted that patient numbers were low in some subgroups.

\section{Nested Case-Control Study}

\section{Patients}

In patients with IBD and UC, a higher proportion of patients with VTE were older, had EIMs of IBD, surgery, CV risk, inherited coagulation disorders, history of VTE, malignancy, and were undergoing major surgery, compared with patients
Fig. 2 Incidence of VTE in patients with a IBD, b UC, and c CD by treatment history in the retrospective cohort study. 5-ASA 5-aminosalicylates, $C D$ Crohn's disease, $C I$ confidence interval, $I B D$ inflammatory bowel disease, $I R$ incidence rate (unique patients with events per 100 patient-years), $N$ number of patients in the analysis population, $n$ number of patients in the category, $T N F i$ tumor necrosis factor inhibitors, $U C$ ulcerative colitis, VTE venous thromboembolism
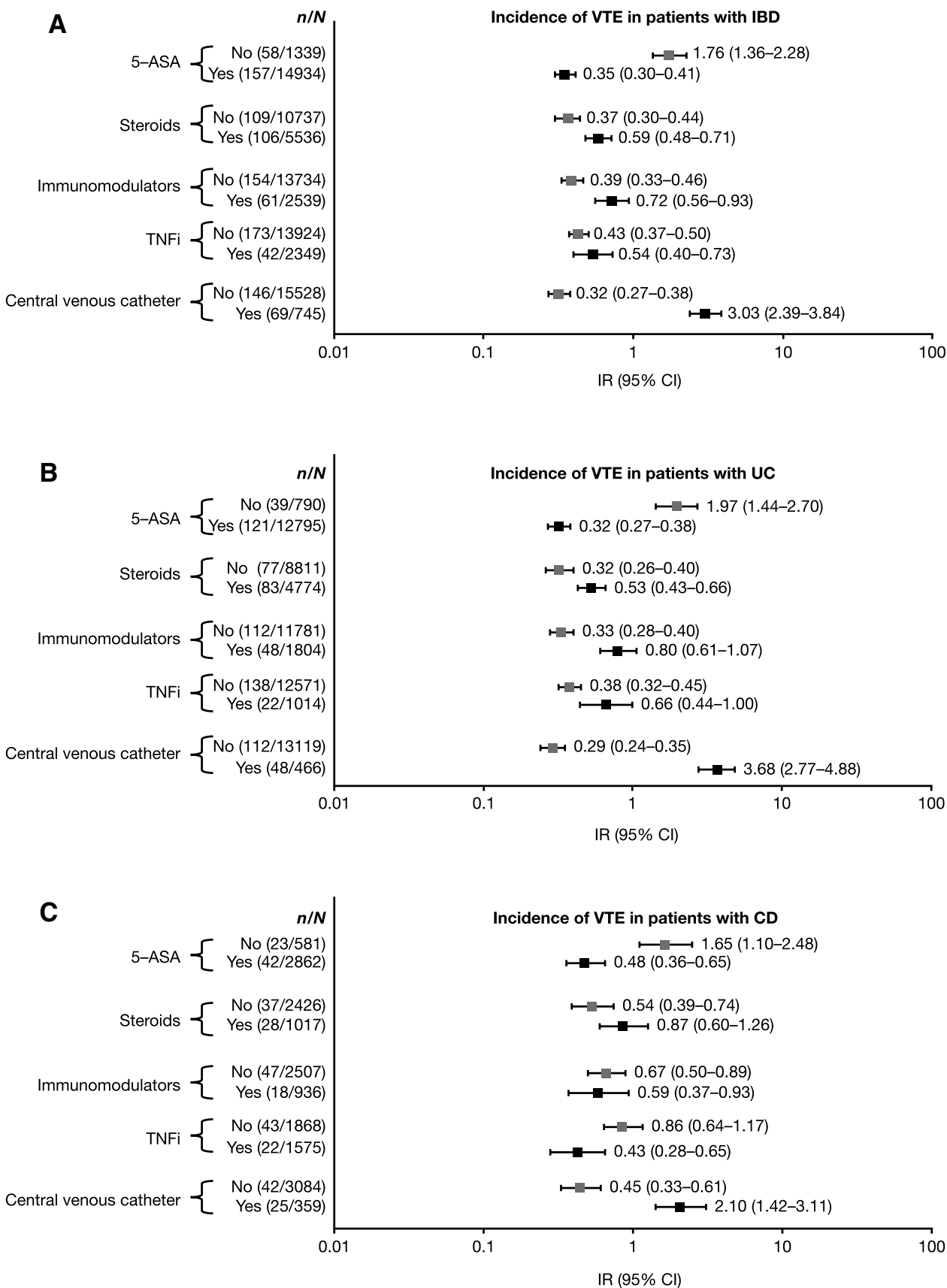
Table 2 Baseline clinical variables in patients with IBD, $\mathrm{UC}$, and $\mathrm{CD}$ with and without VTE in the nested case-control study

\begin{tabular}{|c|c|c|c|c|c|c|}
\hline \multirow[t]{3}{*}{ Variable, $n(\%)$} & \multicolumn{2}{|l|}{ IBD } & \multicolumn{2}{|l|}{$\mathrm{UC}$} & \multicolumn{2}{|l|}{$\mathrm{CD}$} \\
\hline & With VTE & Without VTE & With VTE & Without VTE & With VTE & Without VTE \\
\hline & $N=215$ & $N=860$ & $N=160$ & $N=640$ & $N=65$ & $N=260$ \\
\hline \multicolumn{7}{|l|}{ Sex } \\
\hline Male & $142(66.0)$ & $590(68.6)$ & $98(61.3)$ & 407 (63.6) & $52(80.0)$ & $193(74.2)$ \\
\hline Female & $73(34.0)$ & $270(31.4)$ & $62(38.8)$ & $233(36.4)$ & $13(20.0)$ & $67(25.8)$ \\
\hline \multicolumn{7}{|l|}{ Age, years } \\
\hline$\leq 39$ & $70(32.6)$ & $387(45.0)$ & $45(28.1)$ & $263(41.1)$ & $30(46.2)$ & $137(52.7)$ \\
\hline $40-59$ & $116(54.0)$ & 418 (48.6) & $86(53.8)$ & $337(52.7)$ & $35(53.8)$ & $117(45.0)$ \\
\hline$\geq 60$ & $29(13.5)$ & $55(6.4)$ & $29(18.1)$ & $40(6.3)$ & $0(0.0)$ & $6(2.3)$ \\
\hline \multicolumn{7}{|l|}{ EIMs of IBD } \\
\hline No & 204 (94.9) & 845 (98.3) & $151(94.4)$ & $630(98.4)$ & 63 (96.9) & $254(97.7)$ \\
\hline Yes & $11(5.1)$ & $15(1.7)$ & $9(5.6)$ & $10(1.6)$ & $2(3.1)$ & $6(2.3)$ \\
\hline \multicolumn{7}{|l|}{ Surgery } \\
\hline No & 174 (80.9) & $856(99.5)$ & $129(80.6)$ & 633 (98.9) & 55 (84.6) & $252(96.9)$ \\
\hline Yes & $41(19.1)$ & $4(0.5)$ & $31(19.4)$ & $7(1.1)$ & $10(15.4)$ & $8(3.1)$ \\
\hline \multicolumn{7}{|l|}{$\mathrm{CV}$ risk $(\mathrm{i})^{\dagger}$} \\
\hline No & $97(45.1)$ & $624(72.6)$ & $64(40.0)$ & 465 (72.7) & $36(55.4)$ & $193(74.2)$ \\
\hline Yes & $118(54.9)$ & $236(27.4)$ & $96(60.0)$ & $175(27.3)$ & 29 (44.6) & $67(25.8)$ \\
\hline \multicolumn{7}{|l|}{$\mathrm{CV}$ risk (ii) } \\
\hline No & $107(49.8)$ & $638(74.2)$ & $72(45.0)$ & 473 (73.9) & $38(58.5)$ & $198(76.2)$ \\
\hline Yes & $108(50.2)$ & $222(25.8)$ & $88(55.0)$ & $167(26.1)$ & $27(41.5)$ & $62(23.8)$ \\
\hline \multicolumn{7}{|l|}{$\mathrm{CV}$ risk (iii) ${ }^{\S}$} \\
\hline No & $164(76.3)$ & $817(95.0)$ & $118(73.8)$ & $614(95.9)$ & $53(81.5)$ & $247(95.0)$ \\
\hline Yes & $51(23.7)$ & $43(5.0)$ & $42(26.3)$ & $26(4.1)$ & $12(18.5)$ & $13(5.0)$ \\
\hline \multicolumn{7}{|c|}{ Inherited coagulation disorders } \\
\hline No & $212(98.6)$ & $859(99.9)$ & $158(98.8)$ & $640(100.0)$ & $64(98.5)$ & $260(100.0)$ \\
\hline Yes & $3(1.4)$ & $1(0.1)$ & $2(1.3)$ & $0(0.0)$ & $1(1.5)$ & $0(0.0)$ \\
\hline \multicolumn{7}{|l|}{ History of VTE } \\
\hline No & $200(93.0)$ & $857(99.7)$ & $148(92.5)$ & $639(99.8)$ & $59(90.8)$ & $260(100.0)$ \\
\hline Yes & $15(7.0)$ & $3(0.3)$ & $12(7.5)$ & $1(0.2)$ & $6(9.2)$ & $0(0.0)$ \\
\hline \multicolumn{7}{|c|}{ Hormone therapy ${ }^{\mathbb{I}}$} \\
\hline No & $215(100.0)$ & $860(100.0)$ & $160(100.0)$ & $640(100.0)$ & $65(100.0)$ & $260(100.0)$ \\
\hline Yes & $0(0.0)$ & $0(0.0)$ & $0(0.0)$ & $0(0.0)$ & $0(0.0)$ & $0(0.0)$ \\
\hline \multicolumn{7}{|l|}{ Malignancy } \\
\hline No & $140(65.1)$ & 779 (90.6) & $100(62.5)$ & $577(90.2)$ & $48(73.8)$ & $225(86.5)$ \\
\hline Yes & $75(34.9)$ & $81(9.4)$ & $60(37.5)$ & $63(9.8)$ & $17(26.2)$ & $35(13.5)$ \\
\hline \multicolumn{7}{|c|}{ Undergoing major surgery } \\
\hline No & $100(46.5)$ & $825(95.9)$ & $79(49.4)$ & $611(95.5)$ & $24(36.9)$ & $230(88.5)$ \\
\hline Yes & $115(53.5)$ & $35(4.1)$ & $81(50.6)$ & $29(4.5)$ & $41(63.1)$ & $30(11.5)$ \\
\hline
\end{tabular}

${ }^{\dagger} \mathrm{CV}$ risk (i): hypertension, high-density lipoprotein, diabetes mellitus, history of coronary artery disease, heart failure

${ }^{\ddagger} \mathrm{CV}$ risk (ii): hypertension, high-density lipoprotein, diabetes mellitus

${ }^{\S} \mathrm{CV}$ risk (iii): history of coronary artery disease, heart failure

"Hormone therapy: oral contraceptive, hormone replacement therapy

$C D$ Crohn's disease, $C V$ cardiovascular, EIM extraintestinal manifestation, IBD inflammatory bowel disease, $N$ number of patients in the analysis population, $n$ number of patients in the category, $U C$ ulcerative colitis, VTE venous thromboembolism 

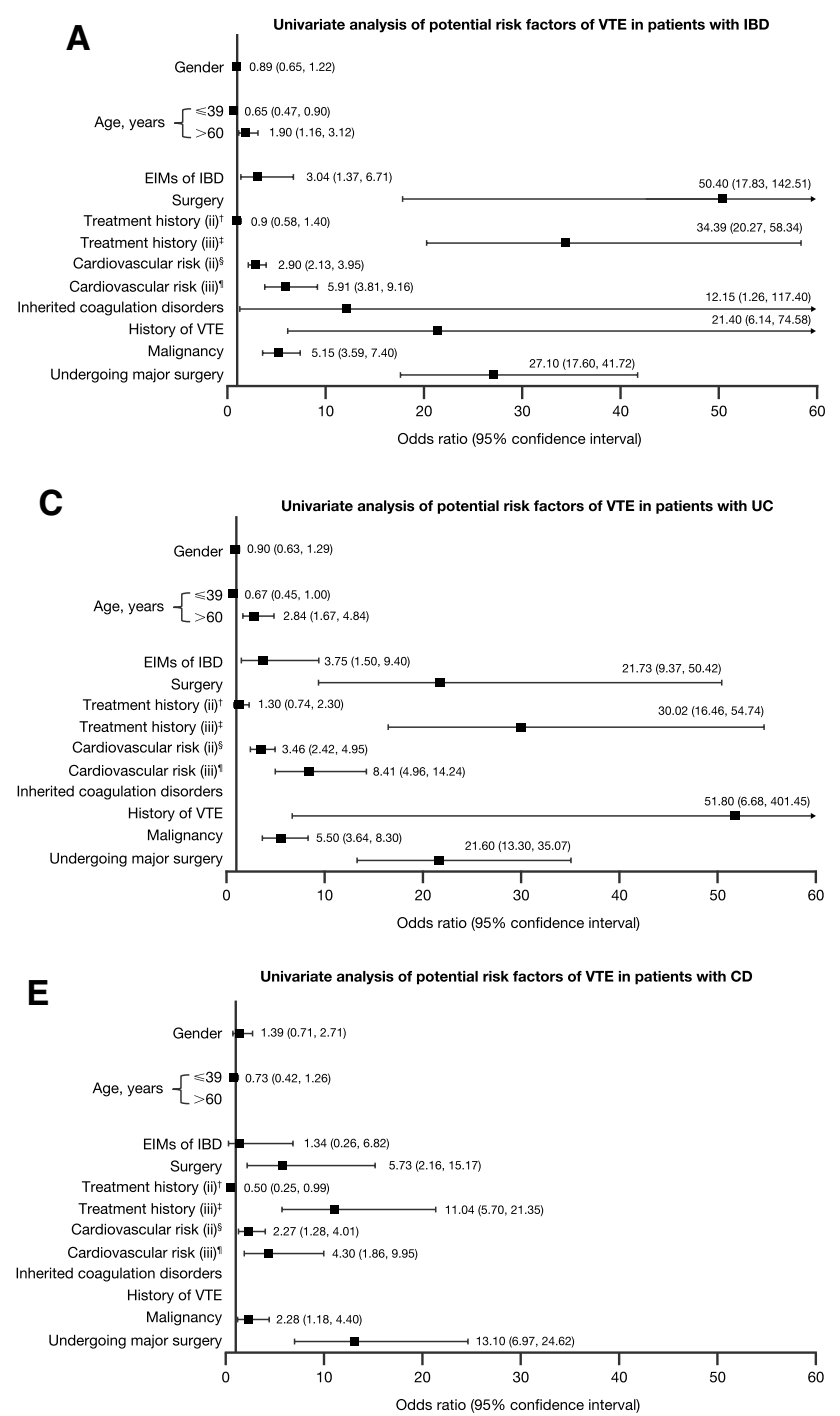

Fig. 3 Univariate and multivariate analysis in patients with a, b IBD, c, $\mathbf{d}$ UC, and e, $\mathbf{f ~ C D}$, of potential risk factors for VTE in the nested case-control study. Reference category for sex: male; reference category for age: 40-59 years; reference category for all other categories: no. ${ }^{\dagger}$ Treatment history (ii): steroids. ${ }^{\ddagger}$ Treatment history (iii):

without VTE. In patients with $\mathrm{CD}$, a higher proportion of patients with VTE had surgery, CV risk, history of VTE, malignancy and were undergoing major surgery, compared with patients without VTE (Table 2).

\section{Univariate and Multivariate Analysis of Potential Risk Factors for VTE}

Univariate analysis was performed for the IBD, UC, and $\mathrm{CD}$ cohorts. Multivariate analysis was then performed for the variables with $\mathrm{OR} \leq 0.67$ or $\geq 1.5$ (point estimation) in the univariate analysis. In the IBD cohort, treatment history
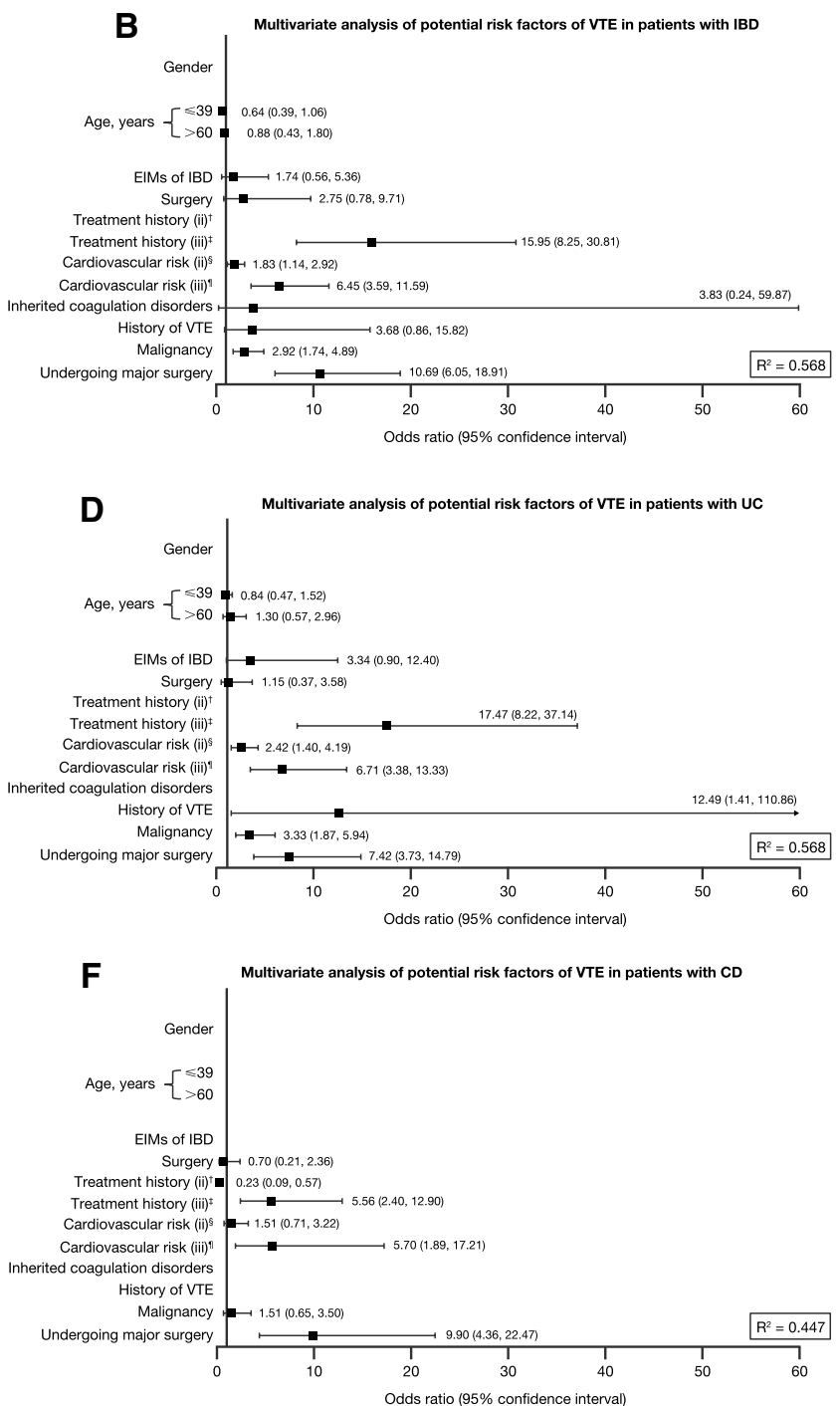

immunomodulators. ${ }^{\S} \mathrm{CV}$ risk (ii): hypertension, high-density lipoprotein, diabetes mellitus. ${ }^{\mathbb{T}} \mathrm{CV}$ risk (iii): history of coronary artery disease, heart failure. $C D$ Crohn's disease, $C I$ confidence interval, $E I M$ extraintestinal manifestation, $I B D$ inflammatory bowel disease, $O R$ odds ratio, $U C$ ulcerative colitis, VTE venous thromboembolism

(immunomodulators), CV risk, malignancy, and undergoing major surgery were identified as potential risk factors for VTE in the multivariate analysis (lower limit of 95\% $\mathrm{CI}$ exceeded risk ratio of 1.0). In the UC cohort, treatment history (immunomodulators), CV risk, history of VTE, malignancy, and undergoing major surgery were identified as potential risk factors for VTE in the multivariate analysis. In the CD cohort, treatment history (immunomodulators), history of coronary artery disease or heart failure, and undergoing major surgery were identified as potential risk factors for VTE in the multivariate analysis (Fig. 3). 


\section{Discussion}

In our study, which was the first analysis conducted using a health insurance claims database in Japan, the IRs of VTE were $0.45,0.40$, and 0.64 in patients with IBD, UC, and CD, respectively, which were slightly higher than those previously reported in Western studies [14, 15]. In contrast with this, the IRs of VTE in the general Asian population have been found to be lower than those in the general Western population [19]. This suggests that Asian patients with IBD are at high risk of VTE, and that establishing a therapeutic strategy for IBD-associated VTE in the Asian population should therefore be made a priority.

While the present study showed a high incidence of IBD-associated VTE, a previous nationwide study in Taiwan identified a low IR of VTE (0.12) in the IBD cohort [18]. As this study is larger than the previous one, it may be more representative of the overall Asian population with IBD. Alternatively, differences between Asian countries with respect to environmental and genetic factors may possibly influence the incidence of IBD-associated VTE events. Additionally, our study does not correlate with our previous findings in Japan, where incidence of VTE was significantly higher in patients with UC versus patients with CD [17]. We hypothesized that these differences in VTE incidence between patients with $\mathrm{UC}$ and patients with $\mathrm{CD}$ in our previous study in Japanese patients may have been due to more systemic inflammation caused by colonic disease in patients with UC [6], and/or may have been due to the higher proportion of patients with UC receiving prednisolone, since they were in a tertiary hospital [20]. A similar finding of a higher IR of VTE in patients with $\mathrm{CD}(0.21)$ versus patients with UC (0.09) was observed in a study in Taiwan [18]. Furthermore, differences in baseline characteristics were observed between patients with UC and patients with CD in this study, with patients with UC having a higher mean age and a higher proportion being female. Although disease activity could not be established from the data collected within the claims database, a difference in the proportion of patients with VTE was observed based on hospitalization status, with higher proportion of VTE events among inpatients versus outpatients, suggesting that high disease activity among inpatients was associated with a high VTE rate. It is of note that in patients with UC, a higher proportion of patients with VTE was observed among inpatients with a treatment history of steroids, immunomodulators, and TNFi compared with inpatients without a treatment history of these therapies (data not shown). Furthermore, a higher proportion of patients without 5-ASA use had VTE events versus patients with 5-ASA use, regardless of hospitalization status (data not shown).
Multivariate analysis identified treatment history (immunomodulators), $\mathrm{CV}$ risk, malignancy, and undergoing major surgery as risk factors for VTE in patients with IBD and UC. Additionally, a history of VTE was identified as a risk factor for VTE in patients with UC. Treatment history (immunomodulators), history of coronary artery disease or heart failure, and undergoing major surgery were identified as risk factors for VTE in patients with CD. Monitoring of patients with IBD, UC, or CD who have these risk factors should be performed to enable rapid detection of VTE events. While these risk factors for VTE have been reported in previous studies [21-24], other risk factors have also been identified. For example, a single-hospital study in Japan identified older age as a risk factor for VTE in patients with IBD [17], while the present study did not. The JMDC claims database does not include elderly patients ( $\geq 75$ years) and contains few patients $\geq 65$ years, which may be why age was not found to be a significant risk factor in the present study, even though elderly patients with IBD have been reported to be relatively rare in Japan (with $<10 \%$ of patients $>65$ years of age) [25]. Furthermore, a single-hospital study in the USA identified that the majority of VTE events in patients with IBD occurred in the outpatient setting [26], while the present study did not. This may be due to differences in therapeutic strategy - including criteria for hospitalization — between Japan and the USA.

Limitations of this study included the selection bias related to the definition of UC, the information bias of VTE outcomes, the lack of precise data reporting medication exposure, and that some misclassification of events may have occurred. The temporary nature of diagnoses based on claims data should also be considered, given that a patient could be diagnosed with VTE, and analyzed as such, on the basis of an insurance claim. This may partly account for the higher prevalence of VTE reported in this analysis compared with other patient populations. Conversely, mild to moderate cases of DVT that did not require treatment may have been missed in this analysis. Furthermore, JMDC claims database data are limited to large, corporate, health insurance unions, and do not include national health insurance subscribers or government public expenditure data. The JMDC claims database only contains limited clinical information; for example, disease severity data are not available, and data are more skewed in sex and age than in the overall Japanese IBD population. In addition, body mass index was not included in the claims data, hormone therapy was not separated into monotherapy or combined therapy, and the severity of heart failure could not be accurately identified. History of VTE was only assessed from 12 months prior to index date. Although a longer period prior to index date to assess the history of VTE would be preferable, this would reduce the sample size available. Therefore, it needs to be 
determined how comparable these results are with the general IBD population.

In conclusion, the incidence of VTE in Japanese patients with IBD, UC, and CD $(0.45,0.40$, and 0.64 , respectively) using the JMDC claims database was slightly higher than that previously reported in Western countries, illustrating that a therapeutic strategy for IBD-associated VTE in the Asian population should promptly be established. Treatment history (immunomodulators), CV risk, malignancy, and undergoing major surgery were identified as risk factors for VTE in Japanese patients with IBD in our study, with similar risk factors reported for patients with $\mathrm{UC}$ and $\mathrm{CD}$.

Supplementary Information The online version contains supplementary material available at https://doi.org/10.1007/s10620-022-07388-w.

Acknowledgment This work was sponsored by Pfizer Japan Inc, Tokyo, Japan. All analyses were performed by A2 Health Care. Medical writing support, under the guidance of the authors, was provided by Pauline Craig, PhD, CMC Connect, McCann Health Medical Communications and was funded by Pfizer Japan Inc, Tokyo, Japan, in accordance with Good Publication Practice (GPP3) guidelines (Ann Intern Med 2015;163:461-464).

Author's contributions All authors contributed to the study conception and design. Material preparation and data analyses were performed by N.I., S.H., and T.K. All authors commented on previous versions of the manuscript and approved the version to be published.

Funding This work was sponsored by Pfizer Japan Inc, Tokyo, Japan.

\section{Declarations}

Conflict of interest M.F. has received grants from Fuji Chemical Industries Co., Ltd., JIMRO Co., Ltd., Kamui Pharma Inc., Nobelpharma Co., Ltd., and Yakult Honsha Co., Ltd.; grants and personal fees from AbbVie Inc, AYUMI Pharmaceutical Corporation, BristolMyers Squibb, Daiichi Sankyo Co., Ltd., EA Pharma Co., Ltd., Janssen Pharmaceutical K.K., Kyorin Pharmaceutical Co., Ltd., Mitsubishi Tanabe Pharma Corporation, Mochida Pharmaceutical Co., Ltd., Nippon Kayaku Co., Ltd., Pfizer Inc, Takeda Pharmaceutical Co., Ltd., and ZERIA Pharmaceutical Co., Ltd.; and personal fees from Aspen Japan K.K., AstraZeneca.co.jp, Boehringer Ingelheim GmbH, Celltrion Healthcare Japan Co., Ltd., Chugai Pharmaceutical Co., Ltd., Horii Pharmaceutical Ind., Ltd., Kissei Pharmaceutical Co., Ltd., Medical Tribune, Inc., Nobelpharma Co., Ltd., Olympus Co., Ltd., Sanofi K.K., Shionogi \& Co., Ltd., Taiho Pharmaceutical Co., Ltd., Technical Information Institute Co., Ltd., and Teijin Pharma Ltd. T.K. is a former employee and stockholder of Pfizer Inc, and is a current employee of Eli Lilly and Company. S.A., S.H., F.M., and S.Y. are employees and stockholders of Pfizer Inc. N.I. and N.Y. are former employees and stockholders of Pfizer Inc. M.N. has received lecture fees from Daiichi Sankyo Co., Ltd. K.M. has received grants and personal fees from AbbVie Inc, EA Pharma Co., Ltd., Kissei Pharmaceutical Co., Ltd., Kyorin Pharmaceutical Co., Ltd., Mitsubishi Tanabe Pharma Corporation, Mochida Pharmaceutical Co., Ltd., Nipponn Kayaku Co., Ltd., Pfizer Inc, Sekisui Medical Co., Ltd., Takeda Pharmaceutical Co., Ltd.; and personal fees from Alfresa Pharma Corporation, Gilead Sciences, Inc., GlaxoSmithKline plc, Janssen Pharmaceutical K.K., JIMRO Co., Ltd., MIYARISAN Pharmaceutical Co., Ltd., Thermo Fisher Scientific Inc., and ZERIA Pharmaceutical Co., Ltd.
Open Access This article is licensed under a Creative Commons Attribution-NonCommercial 4.0 International License, which permits any non-commercial use, sharing, adaptation, distribution and reproduction in any medium or format, as long as you give appropriate credit to the original author(s) and the source, provide a link to the Creative Commons licence, and indicate if changes were made. The images or other third party material in this article are included in the article's Creative Commons licence, unless indicated otherwise in a credit line to the material. If material is not included in the article's Creative Commons licence and your intended use is not permitted by statutory regulation or exceeds the permitted use, you will need to obtain permission directly from the copyright holder. To view a copy of this licence, visit $\mathrm{http} / / /$ creativecommons.org/licenses/by-nc/4.0/.

\section{References}

1. McDowell C, Farooq U, Haseeb M. Inflammatory bowel disease (IBD). In: StatPearls [Internet], Treasure Island, FL; StatPearls Publishing;2020.

2. Asakura K, Nishiwaki Y, Inoue N, Hibi T, Watanabe M, Takebayashi T. Prevalence of ulcerative colitis and Crohn's disease in Japan. J Gastroenterol. 2009;44:659-665.

3. Murakami Y, Nishiwaki Y, Oba MS et al. Estimated prevalence of ulcerative colitis and Crohn's disease in Japan in 2014: an analysis of a nationwide survey. J Gastroenterol. 2019;54:1070-1077.

4. Yuhara H, Steinmaus C, Corley D et al. Meta-analysis: the risk of venous thromboembolism in patients with inflammatory bowel disease. Aliment Pharmacol Ther. 2013;37:953-962.

5. Saibeni S, Saladino V, Chantarangkul V et al. Increased thrombin generation in inflammatory bowel diseases. Thromb Res. 2010;125:278-282.

6. Nguyen GC, Sam J. Rising prevalence of venous thromboembolism and its impact on mortality among hospitalized inflammatory bowel disease patients. Am J Gastroenterol. 2008;103:2272-2280.

7. Office of the Surgeon General (US), National Heart Lung and Blood Institute (US). INTRODUCTION: Definitions of deep vein thrombosis and pulmonary embolism. In: The Surgeon General's call to action to prevent deep vein thrombosis and pulmonary embolism, Rockville, MD; Office of the Surgeon General (US);2008.

8. Włodarczyk M, Sobolewska A, Fichna J, Wiśniewska-Jarosińska M. Prevention and therapeutic strategies of thromboembolic events in patients with inflammatory bowel diseases: a report of three cases. Curr Drug Targets. 2015;16:194-198.

9. Alatri A, Schoepfer A, Fournier N et al. Prevalence and risk factors for venous thromboembolic complications in the Swiss Inflammatory Bowel Disease Cohort. Scand J Gastroenterol. 2016;51:1200-1205.

10. Purnak T, Yuksel O. Overview of venous thrombosis in inflammatory bowel disease. Inflamm Bowel Dis. 2015;21:1195-1203.

11. Grainge MJ, West J, Card TR. Venous thromboembolism during active disease and remission in inflammatory bowel disease: a cohort study. Lancet. 2010;375:657-663.

12. Harbord M, Annese V, Vavricka SR et al. The first European evidence-based consensus on extra-intestinal manifestations in inflammatory bowel disease. J Crohns Colitis. 2016;10:239-254.

13. White RH, Keenan CR. Effects of race and ethnicity on the incidence of venous thromboembolism. Thromb Res. 2009;123:S11-17.

14. Bernstein CN, Blanchard JF, Houston DS, Wajda A. The incidence of deep venous thrombosis and pulmonary embolism among 
patients with inflammatory bowel disease: a population-based cohort study. Thromb Haemost. 2001;85:430-434.

15. Kappelman MD, Horvath-Puho E, Sandler RS et al. Thromboembolic risk among Danish children and adults with inflammatory bowel diseases: a population-based nationwide study. Gut. 2011;60:937-943.

16. Sonoda K, Ikeda S, Mizuta Y, Miyahara Y, Kohno S. Evaluation of venous thromboembolism and coagulation-fibrinolysis markers in Japanese patients with inflammatory bowel disease. J Gastroenterol. 2004;39:948-954.

17. Ando K, Fujiya M, Nomura $Y$ et al. The incidence and risk factors of venous thromboembolism in Japanese inpatients with inflammatory bowel disease: a retrospective cohort study. Intest Res. 2018;16:416-425.

18. Weng MT, Park SH, Matsuoka K et al. Incidence and risk factor analysis of thromboembolic events in East Asian patients with inflammatory bowel disease, a multinational collaborative study. Inflamm Bowel Dis. 2018;24:1791-1800.

19. Lee LH, Gallus A, Jindal R, Wang C, Wu CC. Incidence of venous thromboembolism in Asian populations: a systematic review. Thromb Haemost. 2017;117:2243-2260.

20. Sarlos P, Szemes K, Hegyi P et al. Steroid but not biological therapy elevates the risk of venous thromboembolic events in inflammatory bowel disease: a meta-analysis. J Crohns Colitis. 2018;12:489-498.
21. Rocha AT, Paiva EF, Lichtenstein A, Milani R Jr, Cavalheiro CF, Maffei FH. Risk-assessment algorithm and recommendations for venous thromboembolism prophylaxis in medical patients. Vasc Health Risk Manag. 2007;3:533-553.

22. Alkim H, Koksal AR, Boga S, Sen I, Alkim C. Etiopathogenesis, prevention, and treatment of thromboembolism in inflammatory bowel disease. Clin Appl Thromb Hemost. 2017;23:501-510.

23. Ageno W, Becattini C, Brighton T, Selby R, Kamphuisen PW. Cardiovascular risk factors and venous thromboembolism: a metaanalysis. Circulation. 2008;117:93-102.

24. Eichinger S, Pecheniuk NM, Hron G et al. High-density lipoprotein and the risk of recurrent venous thromboembolism. Circulation. 2007;115:1609-1614.

25. Mahlich J, Matsuoka K, Sruamsiri R. Biologic treatment of Japanese patients with inflammatory bowel disease. BMC Gastroenterol. 2018;18:160.

26. Scoville EA, Konijeti GG, Nguyen DD, Sauk J, Yajnik V, Ananthakrishnan AN. Venous thromboembolism in patients with inflammatory bowel diseases: a case-control study of risk factors. Inflamm Bowel Dis. 2014;20:631-636.

Publisher's Note Springer Nature remains neutral with regard to jurisdictional claims in published maps and institutional affiliations. 